\section{Efetivação da Lei Menino Bernardo pelas redes de proteção e de atendimento a crianças e adolescentes}

\author{
Enforcement of the Menino Bernardo Law on \\ Networks for the Protection and Care of Children \\ and Adolescents in Brazil
}

\section{Cumplimiento de la Ley Menino Bernardo por parte de las redes de protección y atención a niños y adolescentes}

Adalberto de Araújo Trindade 1

Jean Von Hohendorff 2

\title{
Resumo
}

Embora muitos países estejam implementando leis que proíbem o uso de violência física como pretensa prática educativa, a Organização Mundial da Saúde indica que a efetivação dessas leis ainda é um desafio. Objetivou-se, portanto, verificar se as redes de proteção e de atendimento a crianças e adolescentes em situação de risco estão organizadas para efetivar a Lei Menino Bernardo (Lei no 13.010/2014). Por meio de uma abordagem qualitativa e exploratória, foram entrevistados 16 profissionais de diferentes serviços dessas redes de um município do interior do Rio Grande do Sul, Brasil. Os dados coletados nas entrevistas foram analisados por meio da análise temática do tipo indutiva, resultando em quatro temas: (1) prática aceita culturalmente; (2) (des)conhecimento da lei; (3) carências das redes e (4) estratégias de efetivação. Tomados em conjunto, os quatro temas indicam a não efetivação da Lei Menino Bernardo pelas redes de proteção e de atendimento a crianças $e$ adolescentes em situação de risco no município onde a pesquisa foi realizada. Evidenciaram-se, portanto, a aceitação social da violência na educação de crianças e adolescentes, o desconhecimento da Lei Menino Bernardo pelos profissionais e a fragmentação entre os profissionais e os serviços das redes. Diante disso, é necessário aumentar o investimento no fortalecimento de recursos institucionais e em capacitações, visando ao desenvolvimento profissional e à qualificação dos serviços das redes.

Maus-Tratos Infantis; Defesa da Criança e do Adolescente; Educação Infantil; Relações Pais-Filho; Legislação como Assunto

\author{
Correspondência \\ A. A. Trindade \\ Rua Bento Gonçalves 87, apto. 801, Passo Fundo, RS \\ 99020-060, Brasil. \\ adalberto.atd@gmail.com \\ 1 Universidade Federal de Pelotas, Pelotas, Brasil. \\ 2 Faculdade Meridional (IMED), Passo Fundo, Brasil.
}


Ao longo da história, a infância foi compreendida de formas distintas, de acordo com o contexto histórico-cultural de cada época 1,2. Em geral, as crianças eram reconhecidas como pequenos adultos. Suas características desenvolvimentais não eram consideradas, sendo alvos de mão de obra barata e expostas à exploração do trabalho infantil e à violência sexual, uma vez que participavam das mesmas atividades dos adultos, sem qualquer diferenciação 1 . A partir do século XVIII, as concepções modernas da infância foram cristalizadas, tomando caráter distinto e constituindo-se como uma etapa própria do desenvolvimento humano, na sua singularidade 3,4. A adolescência, no entanto, somente passou a ser considerada como uma etapa do desenvolvimento humano, com características distintas da infância e da adultez, após as concepções modernas da infância, entre os séculos XIX e XX 1,5. Nesse sentido, as concepções modernas da infância e da adolescência resultam de processos histórico-culturais 6 .

Essas concepções são inerentes à condição humana, sendo um processo dialético de avanços e retrocessos, e não um processo simples e contínuo de evolução. Questões como desigualdades socioeconômicas, de gênero e de raça exercem influência sobre essas ideias. Coexistem diferentes concepções de infância e de adolescência em um mesmo contexto histórico-cultural, a partir dos diferentes marcadores sociais da diferença ${ }^{7}$. Atualmente, no Brasil, são consideradas crianças aquelas pessoas de até 12 anos incompletos, ao passo que são consideradas adolescentes aquelas entre 12 e 18 anos, idade na qual atingem a maioridade civil 8 .

As mudanças decorrentes da modernidade nas interações parentais têm levantado progressiva indeterminação quanto ao papel dos pais/responsáveis na educação de seus filhos 9 . Essas interações requerem cuidados e estabelecimento de limites. Dessa forma, há um conjunto de recursos emocionais e comportamentais que são chamados de estilos parentais 10. Maccoby \& Martin 11, baseados no modelo de Baumrind 12, propuseram um modelo conceitual de estilos parentais. Por meio das dimensões associadas de responsividade e exigência, os estilos parentais foram classificados em autoritativo, autoritário, indulgente e negligente. Tais estilos compreendem as práticas educativas 10.

As distintas práticas educativas podem ser mais ou menos igualitárias: as indutivas, que indicam à criança a consequência de seus comportamentos, fazendo-a refletir e analisar a situação, e as coercitivas, que compreendem ações controladoras que envolvem ameaças e privações, uso direto da força e de punições físicas. Práticas educativas coercitivas relacionam-se com problemas de comportamento, como agressividade e comportamento opositor, e produzem emoções intensas, como medo, raiva e ansiedade 13,14. Sendo assim, práticas educativas podem ser de caráter protetor ou de risco para o desenvolvimento 10,15. A conscientização sobre as consequências de práticas coercitivas é insuficiente, e ainda persiste o uso da violência, justificada como prática educativa 16.

Práticas educativas coercitivas são consideradas violentas e relacionadas ao desenvolvimento de problemas internalizantes e externalizantes 14 , repetição de comportamentos agressivos na escola 17 , comportamentos antissociais 18 , interações sociais disfuncionais (principalmente no contexto familiar 19), sentimentos como medo, insegurança e baixa autoestima 20 , aumento do risco de desenvolver transtornos mentais, uso abusivo de álcool e de outras drogas 21 , ampla variedade de sintomas psicopatológicos (como sintomas obsessivos, compulsivos e depressivos, incluindo o sofrimento psicológico geral 22) e distúrbios de comunicação (como o mutismo e a gagueira 23). As consequências da exposição à violência na infância e na adolescência, ou seja, a práticas educativas coercitivas, podem perdurar até a idade adulta 15,22. São associadas, ainda, a consequências comunitárias e sociais, acarretando problemas ocupacionais, que podem impactar o desenvolvimento social e econômico de um país. Devido a isso, a violência contra crianças e adolescentes é considerada um problema de saúde pública 24 .

Esse tipo de violência ocorre em todo o mundo, em diferentes culturas e de diversas formas 25 . Há poucas décadas, o fenômeno tem adquirido relevância social, sendo alvo de enfrentamento nas esferas pública e privada 26 . Mundialmente, um em cada quatro adultos sofreu violência física quando criança. Em 2016, 23\% das crianças de todo o mundo sofreram violência física, 36\% das crianças passaram por algum tipo de violência emocional, $16 \%$ por negligência e $26 \%$ sofreram violência sexual 24 . No Brasil, ainda não se encontram dados gerais de prevalência da violência contra crianças e adolescentes sistematizados, uma vez que não são todos os casos que são devidamente notificados 27,28 . A falta de sistematização das informações e de ferramentas padronizadas de notificação, aliada ao despreparo de profissionais, contribui para a subnotificação de casos 29 . Apesar disso, um estudo de meta-análise indica que o Brasil possui as maiores taxas estimadas de violência contra crianças e adolescentes em 
todo o mundo 30. Em outro estudo realizado no Brasil, cujo objetivo foi levantar a prevalência das distintas formas de violência sofridas por crianças e adolescentes, a violência física foi a mais prevalente (85\%), seguida da violência emocional 31.

No Brasil, crianças e adolescentes são considerados sujeitos de direito 2. A partir da promulgação da Constituição Federal, a defesa dos direitos fundamentais de crianças e adolescentes é legalmente amparada. De acordo com o Artigo 227 da Constituição Federal 32, "é dever da família, da sociedade e do Estado assegurar à criança e ao adolescente, com absoluta prioridade, o direito à vida, à saúde, à alimentação, à educação, ao lazer, à profissionalização, à cultura, à dignidade, ao respeito, à liberdade, à convivência familiar e comunitária...”. Em 1990, o Brasil ratificou a Convenção sobre os Direitos da Criança, adotada pela Assembleia Geral da Organização das Nações Unidas (ONU) em 1989 33. Trata-se do instrumento de direitos humanos reconhecido por 196 países na história universal. Em seu Artigo no 19 34, declara que "os Estados Partes devem adotar todas as medidas legislativas, administrativas, sociais e educacionais apropriadas para proteger a criança contra todas as formas de violência física ou mental, ofensas ou abusos, negligência ou tratamento displicente, maus-tratos ou exploração, inclusive abuso sexual, enquanto a criança estiver sob a custódia dos pais, do tutor legal ou de qualquer outra pessoa responsável por ela”.

A diligência de que crianças e adolescentes estão em uma etapa peculiar de desenvolvimento e que requerem proteção integral tem seu marco legal a partir da promulgação da Lei no 8.069 de 1990, conhecida como Estatuto da Criança e do Adolescente (ECA) 8. Surge, então, uma nova concepção de infância e de adolescência, na qual a criança e o/a adolescente são sujeitos em etapa peculiar de desenvolvimento, de direitos civis, sociais e humanos, necessitando de cuidados especiais e de proteção integral 8. Assume, então, caráter preventivo, coibindo a violação de seus direitos fundamentais 35 . Porém, essa concepção de infância e de adolescência não é totalmente compartilhada pela sociedade 36 .

O ECA 8 dispõe sobre a proteção integral, tendo por objetivo a defesa dos direitos de crianças e adolescentes: "a criança e o/a adolescente gozam de todos os direitos fundamentais inerentes à pessoa humana (...), assegurando-lhes (...) o desenvolvimento físico, mental, moral, espiritual e social, em condições de liberdade $e$ de dignidade". O Artigo 17 preconiza o direito ao respeito, que "consiste na inviolabilidade da integridade física, psíquica e moral da criança e do adolescente (...), sendo dever de todos velar pela sua dignidade, pondo-os a salvo de qualquer tratamento desumano, violento, aterrorizante, vexatório ou constrangedor" (Artigo 18). Além disso, prevê medidas de proteção quando houver ameaça ou violação de seus direitos. Isto é, em casos de suspeita ou confirmação de violência contra crianças e adolescentes, o ECA estabelece a notificação obrigatória (Artigo 13). Ainda prevê infrações administrativas (multa) caso médicos, professores ou responsáveis por estabelecimento de atenção à saúde e de Ensino Fundamental, pré-escola ou creche deixem de notificar à autoridade competente os casos de que tenham conhecimento (Artigo 245) 8.

Em atenção ao impacto na morbimortalidade da população causado pela violência, em 2001 foi aprovada a Política Nacional de Redução da Morbimortalidade por Acidentes e Violências 37. Nela, é indicado que a violência contra crianças e adolescentes é uma causa significativa de morbimortalidade nesse grupo, sendo alvo prioritário de atenção. Contudo, o fenômeno é considerado um desafio para o setor de saúde, uma vez que fatores culturais e a falta de conhecimento de usuários e profissionais dos serviços são apontados como dificultadores de enfrentamento.

Nas últimas décadas, leis complementares têm sido integradas ao ECA. Em 26 de junho de 2014, foi promulgada a Lei no 13.010 38, cujo objetivo é estabelecer "o direito da criança e do adolescente de serem educados e cuidados sem o uso de castigos físicos ou de tratamento cruel ou degradante”. Popularmente reconhecida pelo Projeto de Lei no 7.672/2010 como Lei da Palmada, após promulgada, foi chamada de Lei Menino Bernardo, em alusão ao caso de Bernardo Uglione Boldrini (http://naobataeduque.org.br/ caso-do-menino-bernardo-o-brasil-precisa-ouvir-as-suas-criancas/). Trata-se de uma lei que prevê sanções administrativas àqueles que maltratarem crianças e adolescentes (como encaminhamento a programa oficial ou comunitário de proteção à família e/ou a tratamento psicológico ou psiquiátrico). Nela, constam também delineadas as políticas públicas e medidas para coibir a violência contra crianças e adolescentes 38 .

A ONU 39 recomenda legislações específicas que proíbam legalmente o uso de práticas educativas coercitivas. Nesse sentido, a Lei Menino Bernardo emerge como uma tentativa de o Brasil atualizar seu marco legal em conformidade com os padrões do direito internacional 40. Contudo, o relatório mundial sobre prevenção à violência de 2014, que inclui dados de 133 países, entre eles o Brasil, evidencia que frequentemente leis promulgadas que visam à proibição de práticas educativas coercitivas não são 
efetivadas adequadamente. Dos $76 \%$ dos países que promulgaram tais leis, apenas 30\% atingiram sua efetivação plena 41 . A partir disso, o objetivo desta pesquisa foi verificar se as redes de proteção e de atendimento a crianças e adolescentes em situação de risco estão organizadas para efetivar a referida lei em um município do interior do Rio Grande do Sul, Brasil.

\section{Método}

\section{Delineamento}

Foi realizada uma pesquisa qualitativa, de cunho exploratório e de corte transversal.

\section{Participantes}

Participaram da pesquisa 16 profissionais que atuavam nos serviços das redes de proteção (10) e de atendimento (6) a crianças e adolescentes em situação de risco de um município do interior do Rio Grande do Sul; 13 desses profissionais eram mulheres, com idades entre 31 e 66 anos (média $\mathrm{M}=44$; desvio padrão - DP = 9,86). A categoria profissional preponderante foi de psicólogas (6); as demais foram assistentes sociais (3), pedagogas (2), enfermeira (1). As ocupações foram de conselheiros tutelares (2), juiz de direito (1) e delegado de polícia (1). O tempo de atuação profissional nos serviços variou entre 4 e 16 anos $(M=7,68 ; \mathrm{DP}=3,62)$. Três participantes haviam realizado formação específica sobre violência contra crianças e adolescentes (pós-graduação lato e stricto sensu), os demais relataram ter participado de eventos e capacitações sobre o tema.

Foram incluídos profissionais que atuavam nos serviços das redes de proteção e de atendimento a crianças e adolescentes em situação de risco, de ambos os gêneros, e com, no mínimo, quatro anos de atividade, tendo em vista o ano (2014) de promulgação da Lei Menino Bernardo. Não houve critérios de exclusão. A quantidade de participantes foi determinada a partir do poder da informação 42 em pesquisas qualitativas. Em síntese, o poder da informação indica que quanto mais informações relevantes ao problema de pesquisa forem coletadas com cada participante, menor será a quantidade necessária de participantes.

\section{Instrumentos}

Os instrumentos utilizados foram desenvolvidos especificamente para esta pesquisa, sendo eles um questionário de dados sociodemográficos e laborais utilizado para caracterizar os participantes, respondido pelo/a próprio/a e preenchido pelo pesquisador, além de um roteiro de entrevista semiestruturada, composto de 12 perguntas.

\section{Procedimentos de coleta e análise de dados}

Após aprovação do Comitê de Ética em Pesquisa (CAAE 03303818.0.0000.5319), foram contatados serviços das redes de proteção e de atendimento a crianças e adolescentes em situação de risco de um município do interior do Rio Grande do Sul para estabelecer parcerias para a coleta de dados. Essas parcerias foram estabelecidas a partir de proposta descrita em estudo anterior 43: (1) levantamento dos serviços; (2) contato telefônico e/ou via e-mail; (3) agendamento de reuniões e (4) formalização da parceria por meio da assinatura do Termo de Concordância. Na tentativa de estabelecer parceria com o Ministério Público, tal órgão justificou a não participação da Promotoria de Justiça de Defesa da Infância e da Juventude pela indisponibilidade de agenda.

Foi solicitado aos gestores dos serviços que indicassem profissionais para a realização da coleta de dados, a partir dos critérios de inclusão adotados. A coleta de dados ocorreu nas dependências dos serviços, de acordo com a preferência do/a participante e mediante seu consentimento, sendo iniciada pelo preenchimento do questionário de dados sociodemográficos e laborais. Em seguida, foi conduzida a entrevista, gravada em áudio e transcrita na íntegra. Foi atribuído um codinome, seguido de um número para cada participante, com o objetivo de preservar seu anonimato. As entrevistas duraram 
entre 10 e 42 minutos, com média de 25 minutos, e foram realizadas entre dezembro de 2018 e março de 2019 pelo primeiro autor, após treinamento prévio com o segundo autor. Com vistas a verificar a adequação do roteiro de entrevista semiestruturada, a primeira teve caráter piloto.

Os dados coletados por meio das entrevistas foram examinados de acordo com a análise temática do tipo indutiva 44, composta de seis etapas: (1) familiarização com os dados por meio da transcrição e revisão das entrevistas; (2) codificação por meio da identificação e seleção de extratos; (3) busca e identificação de temas por meio de revisão e agrupamento dos extratos; (4) revisão dos temas, identificados e agrupados pela similaridade de conteúdo; (5) definição e nomeação final de temas, ilustrados por trechos representativos de análise; (6) relatório e discussão dos resultados. Foi utilizado o software NVivo 12 (https://www.qsrinternational.com/nvivo/home) como auxílio para a análise dos dados coletados pelas entrevistas.

\section{Resultados e discussão}

A análise de dados resultou em quatro temas: (1) prática aceita culturalmente; (2) (des)conhecimento da lei; (3) carências das redes e (4) estratégias de efetivação. Tomados em conjunto, os temas indicam a não efetivação da Lei Menino Bernardo pelas redes de proteção e de atendimento no município onde a pesquisa foi realizada.

O tema prática aceita culturalmente indica que o uso de violência física na educação de crianças e adolescentes é naturalizado pela sociedade, sendo um entrave à efetivação da Lei Menino Bernardo. Alguns participantes refletiram sobre a aceitação social do uso de práticas educativas coercitivas, como mostram os trechos: "Eu observo aqui no trabalho, que as pessoas têm naturalizado a questão da palmada, chinelada, as crianças apanharem, é natural ainda" (P11). A aceitação de profissionais também foi evidenciada: "É uma lei um tanto inócua. Essa 'Lei da Palmada' foi um exagero legislativo" (P9).

A cultura é indicada como influência no uso de práticas educativas coercitivas, ou seja, no uso da violência nas interações parentais estabelecidas 45,46. Os participantes da pesquisa buscaram explicar possíveis causas para a adoção de tais práticas, como a transmissão de modelos parentais aprendidos na infância e o baixo nível socioeconômico da família: "Porque muitos cometem alguns atos só repetindo ações que viveram. Tô vivo, apanhei e não deu nada" (P12); "Às vezes, é uma questão de educação do pai, que recebeu a educação sendo vítima de violência (...); Às vezes, ela pode tá inserida num contexto de falta de acesso a condições, minimamente, dignas de habitação" (P14).

A transmissão de práticas educativas coercitivas é marcada por suas próprias experiências pregressas de educação. As experiências vividas pelos pais em suas famílias de origem são apontadas como fatores de risco para a utilização dessas práticas nas interações com seus filhos 20,45,47. Pais que foram educados por meio da violência tendem a repetir o modelo parental aprendido. Ainda que não seja uma condição determinante, famílias oriundas de baixo nível socioeconômico estão suscetíveis à utilização de práticas educativas coercitivas 48 . Nesse sentido, por meio do acompanhamento e da intervenção dos serviços das redes, as famílias poderão adquirir novos repertórios e habilidades educativas. Contudo, suas necessidades básicas (como saúde, alimentação e moradia) devem ser satisfeitas 49. Os profissionais podem identificar esses fatores de risco e facilitar o acesso aos serviços necessários para fortalecer as famílias e prevenir novos episódios de violência 50.

Mesmo a violência física sendo considerada uma prática aceita culturalmente, os participantes relataram a possibilidade de mudança social com a efetivação da Lei Menino Bernardo: "Eu vejo como uma mudança bem severa em termos de cultura, de pensamento" (P3). Embora as políticas públicas de proteção à infância e a adolescência sofram lentos e progressivos processos de mudanças, espera-se que a cultura se renove por meio da adoção de novas concepções, crenças e hábitos 51 . Nesse sentido, a Lei Menino Bernardo fundamenta-se como um importante instrumento de sensibilização social e a ela é atribuída a função de mudança da cultura brasileira 52 . Apesar disso, existe uma grande dificuldade de incluir as famílias em processos de transformações culturais, constituindo-se como um desafio às redes 53. Há um longo percurso a ser percorrido para tornar efetiva a defesa dos direitos de crianças e adolescentes, como é o direito de serem educados e cuidados sem o uso da violência 46.

Embora a Lei Menino Bernardo preveja campanhas de educação e sensibilização da população, o segundo tema indica o (des)conhecimento da lei. Tal desconhecimento parece estar relacionado ao 
primeiro tema, ou seja, ao fato de a violência física ser uma prática aceita culturalmente. Os/as participantes indicaram o conhecimento do caso Menino Bernardo em detrimento do conhecimento da Lei Menino Bernardo: "Já ouvi falar do caso, mas a lei não" (P6); "A gente sabe da história, mas efetivamente os critérios da lei eu não saberia te dizer" (P13). Dentre aqueles que mencionaram conhecer a Lei Menino Bernardo, percebeu-se o conhecimento superficial: "Já ouvi falar, mas não tenho grande aprofundamento dessa lei” (P8). Após breve explicação sobre a Lei Menino Bernardo, alguns profissionais participantes se referiram a ela utilizando nomenclaturas diversas: "Aquela da Xuxa?” (P13). "A Lei da Palmada já conhecia, mas não sabia que tinha migrado" (P12). Dos 16 profissionais participantes, apenas seis relataram algum conhecimento da Lei Menino Bernardo. O não conhecimento da Lei Menino Bernardo pelos profissionais pode ser considerado o principal dificultador para a sua efetivação, tendo em vista que o desconhecimento impede que suas atuações nos serviços em que estão inseridos sejam guiadas pela Lei Menino Bernardo.

Outro aspecto significativo levantado pelos profissionais participantes desta pesquisa foi o desconhecimento da Lei Menino Bernardo pela população em geral, devido à violência física ser uma prática aceita culturalmente, conforme evidenciado no primeiro tema: "Existe uma resistência social, de entender que a lei não é adequada. Então, a gente teria que fazer um trabalho de conscientização pra vencer essa resistência social, que é decorrente dessa cultura" (P14); "É uma situação cultural da população, é difícil uma família que você atenda que aceite essa lei de bom gosto" (P8). Logo, se há resistência das famílias, limitada ou nula será sua intenção de conhecer a Lei Menino Bernardo. Sequer haverá investimento na adoção de novos repertórios e habilidades educativas.

A Organização Mundial da Saúde (OMS) 41 indica que, em países de todo o mundo, leis que visam à proibição de práticas educativas coercitivas não são plenamente efetivadas. De acordo com os resultados desta pesquisa, a não efetivação de tais leis pode estar relacionada ao seu desconhecimento. Os profissionais participantes desta pesquisa indicaram fatores que podem influenciar o desconhecimento da Lei Menino Bernardo: "Como é uma lei meio recente - 2014, eu acho que ela não atingiu grande parte das pessoas ainda” (P11). Estratégias que podem facilitar o conhecimento da Lei Menino Bernardo foram mencionadas: "A informação, de divulgar. A questão do estudo técnico, a gente teria que estudar, se apropriar pra ver como colocar em prática” (P4). Finalmente, a percepção de necessidade de capacitação foi relatada: "Quanto mais formação, capacitação, profissionalização, atualização específica melhor. O ideal seria ter, inclusive, supervisão técnica para quem atua" (P4).

Os resultados acerca do desconhecimento da lei apontam a importância de aumentar o investimento na capacitação dos profissionais, visando a seu desenvolvimento profissional e à qualificação dos serviços. O desenvolvimento profissional se dá por meio da aprendizagem e da formação continuada e reflete na atuação prática dos profissionais 54. O desenvolvimento profissional pode ser individualizado, ou sua aprendizagem e suas competências podem ser desenvolvidas coletivamente 55 . Em se tratando de profissionais que atuam em serviços públicos, tal desenvolvimento pode ser feito de forma que envolva todas as equipes de trabalho. O investimento de recursos públicos deve fornecer subsídios para a capacitação dos profissionais 56. Oportunidades de desenvolvimento profissional informal devem ser criadas. Trocas de aprendizagem e de experiências devem ser oportunizadas aos profissionais que atuam nos diversos serviços, além de capacitações formais que incluam programas sistematizados de treinamento, desenvolvimento e educação 57. Profissionais com oportunidades de desenvolvimento poderão atuar de forma mais adequada e serão capazes de romper com o isolamento de suas próprias categorias profissionais 56.

A inclusão das abordagens da violência contra crianças e adolescentes é urgente na formação de futuros profissionais. A falta de disciplinas nos cursos de graduação que abordem o fenômeno, principalmente no que tange à naturalização de práticas educativas coercitivas, é evidente. É necessário que os currículos sejam reorganizados, de modo que os futuros profissionais possam adquirir conhecimentos e desenvolver capacidades plenas para atuar em distintos contextos socioculturais. Portanto, é fundamental que haja uma abordagem interdisciplinar e transdisciplinar para que os currículos possam ser revistos para abordar o tema das práticas educativas coercitivas, ou seja, da violência.

No terceiro tema, carências da rede, foram identificadas dificuldades específicas para tornar a Lei Menino Bernardo efetiva. Tais dificuldades se relacionam, principalmente, ao desconhecimento da Lei Menino Bernardo: "Dificulta muito a falta de conhecimento" (P1); ao baixo número de profissionais: "A gente é em poucos técnicos pra cuidar de muitos serviços" (P6); à sobrecarga de trabalho: "A gente se perde 
na correria do dia-a-dia, nas execuções de muitas ações" (P1); à falta de estruturas físicas que comportem os serviços: "A gente não tem estrutura física pra dar conta dessa demanda" (P13). Por fim, a comunicação entre os serviços foi apontada como uma importante dificuldade: "A negligência do profissional em não fazer registros. A falta de registros e a falta de abertura para os profissionais se comunicarem” (P10).

A proteção de crianças e adolescentes e, consequentemente, a efetivação de leis como a Lei Menino Bernardo não dependem exclusivamente dos profissionais das redes. A sociedade como um todo é responsável por tal proteção. O poder executivo, a gestão dos serviços, a sociedade civil organizada, o controle social a partir dos Conselhos de Direitos e as organizações não governamentais desempenham papel fundamental. Sendo assim, os resultados sobre as carências da rede indicam que há necessidade de fortalecimento e de articulação entre os profissionais, os serviços e os demais segmentos, sobretudo quanto à comunicação formal ou informal. Isso confirma resultados de estudo prévio 55 que destaca o fortalecimento da aproximação e da comunicação entre os profissionais dos diferentes serviços das redes, visando a intervenções mais efetivas. A fragmentação entre os profissionais e os serviços, o baixo número de profissionais e a sobrecarga de trabalho são dificuldades que prejudicam o trabalho em rede. Além disso, divergências políticas e de concepções, conflito de papéis entre profissionais e serviços, bem como a rotatividade de profissionais, são dificuldades comuns 58,59. Horizontalidade, corresponsabilidade de trabalho, divisão de recursos e compartilhamento de informações são aspectos que podem facilitar a superação dessas dificuldades. Contudo, por si sós, tais aspectos não garantem efetividade na articulação entre profissionais e serviços 53 .

Dentre as iniciativas tomadas em nível nacional, destaca-se o Sistema de Vigilância de Violências e Acidentes (VIVA), implantado pelo Ministério da Saúde por meio da Portaria no 1.356, de 23 de junho de 2006 60. Especificamente, em relação à vigilância e à prevenção de violências, em 2016, foi publicada a Portaria no 204, de 17 de fevereiro de 2016 61, que universalizou a notificação compulsória de violências para todos os serviços de saúde. No entanto, para que a notificação de casos de violência contra crianças e adolescentes seja realizada, é necessário que os profissionais saibam o que é considerado violência, levando em consideração que, como os resultados desta pesquisa indicam, essa é uma prática aceita culturalmente e que há o desconhecimento da Lei Menino Bernardo.

São urgentes a sensibilização e a conscientização dos profissionais quanto ao seu papel legal e ético na efetivação da Lei Menino Bernardo. O empenho dos profissionais e dos serviços, associado aos demais segmentos, pode resultar em ganhos significativos na prevenção da violência contra crianças e adolescentes. Para tanto, no quarto tema, estratégias de efetivação, foram apontados potenciais mecanismos estratégicos para tornar a Lei Menino Bernardo efetiva. Nomeadamente, destacam-se a maior divulgação e discussão desta lei, programas de intervenção preventivos e programas que visem ao treinamento de pais: "Hoje tá faltando um trabalho que alcance mais a sociedade, a população, nesse aspecto. Porque logo depois da promulgação teve movimentos, mas hoje quais são os movimentos, campanhas que a gente vê? Não vê. Outro ponto é que não tem um trabalho alternativo pra fazer com que os pais entendam que tem alternativas do que fazer" (P10).

Os mecanismos que potencialmente podem efetivar a Lei Menino Bernardo citados pelos participantes desta pesquisa estão previstos no Artigo 70-A, inc. I da Lei Menino Bernardo: "a promoção de campanhas educativas permanentes para a divulgação do direito da criança e do adolescente de serem educados e cuidados sem o uso de castigo físico ou de tratamento cruel ou degradante e dos instrumentos de proteção aos direitos humanos" 38. Percebe-se, portanto, que as estratégias de efetivação indicadas pelos profissionais participantes desta pesquisa são as mesmas previstas pela Lei Menino Bernardo. Diante disso, a não realização de tais campanhas educativas, mesmo depois de transcorridos cinco anos da sua promulgação, evidencia claramente sua não efetivação.

\section{Considerações finais}

A Lei Menino Bernardo entrou em vigor há cinco anos. Os temas resultantes desta pesquisa indicaram a sua não efetivação. A violência física ainda é uma prática aceita culturalmente pela população e por alguns profissionais. Isso parece influenciar no (des)conhecimento da lei, segundo tema identificado nesta pesquisa. É possível afirmar que o (des)conhecimento da lei pelos profissionais é o principal entrave à sua efetivação. Além desse entrave, carências das redes foram citadas, destacando-se o 
baixo número de profissionais, a sobrecarga de trabalho e a falta de comunicação entre profissionais e serviços. Estratégias de efetivação da Lei Menino Bernardo também foram citadas, sendo que estas refletem aquelas previstas na própria lei: campanhas de educação permanente para a população geral, fortalecimento e articulação dos órgãos e serviços e capacitação profissional.

Os resultados da presente pesquisa devem levar em consideração o fato de esta ter sido desenvolvida em um município do interior do Rio Grande do Sul. Buscou-se contemplar todos os órgãos e serviços das redes de proteção e de atendimento. Embora profissionais de diferentes áreas tenham sido acessados, é possível que outros setores (por exemplo, organizações não governamentais, Conselhos de Direito) possam fornecer informações relevantes. Estudos futuros podem ser realizados em outros municípios e com setores não contemplados nesta pesquisa. Embora restritos à realidade do município onde a coleta de dados foi realizada e limitados aos setores envolvidos, os resultados aqui apresentados podem indicar uma tendência nacional em relação à não efetivação da Lei Menino Bernardo.

A Lei Menino Bernardo por si só não mudará o atual cenário de violações de direitos de crianças e adolescentes. Urge transcender concepções de práticas coercitivas como estratégia educativa. Exemplos de viabilidades práticas de execução já em vigor são políticas públicas, como a Primeira Infância Melhor. Trata-se uma política pública ofertada no Estado do Rio Grande do Sul, que prevê uma visita domiciliar semanal, com o objetivo de promover o desenvolvimento integral infantil e fortalecer os vínculos familiares e comunitários. Inspirou o Programa Criança Feliz do Governo Federal e é considerada uma das tecnologias sociais mais consistentes da América Latina 62. Por meio de tais visitas, os profissionais podem desempenhar um importante papel de conscientização e de acompanhamento dessas famílias. Para que isso ocorra, os/as profissionais devem ser devidamente capacitados.

\section{Colaboradores}

A. A. Trindade contribuiu na concepção do projeto, execução da pesquisa, análise de dados e interpretação dos resultados e elaboração do texto. J. V. Hohendorff contribuiu na concepção do projeto, análise de dados, interpretação dos resultados. Os autores aprovaram a versão final do artigo.

\section{Informações adicionais}

ORCID: Adalberto de Araújo Trindade (0000-00017058-2972); Jean Von Hohendorff (0000-00027414-5312).

\section{Agradecimentos}

O estudo foi realizado com apoio da Fundação IMED, por meio de bolsa produtividade concedida ao segundo autor J. V. Hahendorff.

\section{Referências}

1. Ariès P. História social da criança e da família. 2a Ed. Rio de Janeiro: LTC Editora; 1981.

2. Domingos ALSO, Silva FDA. Concepções de criança e infância a partir da legislação brasileira. P@rtes (São Paulo) 2016; 1:1-18.

3. Frota AMMC. Diferentes concepções da infância e adolescência: a importância da historicidade para sua construção. Estud Pesqui Psicol 2007; 7:144-57.

4. Vasconcellos VMR, Sarmento MJ. Infância (in) visível. Araraquara: Junqueira \& Marin Editores; 2007.

5. Kimmel DC, Weiner IB. La adolescencia: una transición del desarollo. Barcelona: Ariel; 1998

6. Alves CF, Siqueira AC. Os direitos da criança e do adolescente na percepção de adolescentes dos contextos urbano e rural. Psicol Ciênc Prof 2013; 33:460-73.

7. Heywood C. Uma história da infância. Porto Alegre: Artmed; 2004.

8. Brasil. Lei no 8.069, de 13 de julho de 1990 . Dispõe sobre o Estatuto da Criança e do Adolescente e dá outras providências. Diário Oficial da União 1990; 16 jul.

9. Cassoni C, Caldana RH. Estilos e práticas educativas parentais: revisão sistemática e crítica da literatura. Saabrücken: Novas Edições Acadêmicas; 2017.

10. Macarini SM, Martins GDF, Minetto MFJ, Vieira ML. Práticas parentais: uma revisão da literatura brasileira. Arq Bras Psicol 2010; 62:119-34. 
11. Maccoby E, Martin J. Socialization in the context of the family: parent-child interaction. In: Hetherington EM, editor. Handbook of child psychology. Socialization, personality, and social development. Chichester: Wiley; 1983. p. 1-101.

12. Baumrind D. Effects of authoritative parental control on child behavior. Child Dev 1966; 37:887-907.

13. Hoffman ML. Power assertion by the parent and its impact on the child. Child Dev 1960; 31:129-43

14. Hoffman ML. Moral, internalization, parental power, and the nature of parent-child interaction. Dev Psychol 1975; 11:228-39.

15. Patias ND, Siqueira AC, Dias ACG. Práticas educativas e intervenção com pais: a educação como proteção ao desenvolvimento dos filhos. Mudanças 2013; 21:29-40.

16. Ceconello AM, De Antoni C, Koller SH. Práticas educativas, estilos parentais e abuso físico no contexto familiar. Psicol Estud 2003; 8(n. esp):45-8.

17. Gomes AFC, Azevêdo AVS. Punição corporal e problemas comportamentais em adolescentes. Contextos Clín 2014; 7:76-85.

18. Gomide PIC. Estilos parentais e comportamento antissocial. In: Del Prette Z, Del Prette A, organizadores. Habilidades sociais, desenvolvimento e aprendizagem: questões conceituais, avaliação e intervenção. Campinas: Alínea; 2003. p. 21-60.

19. Bazon MR, Faleiros JM, Pasian MS. Problematizando a face mais insidiosa da violência contra as crianças: a negligência - conceito(s), características e consequências. In: Ponzilacqua MHP, organizador. Violência doméstica, agressão sexual e direito: da constatação ao enfrentamento pela perspectiva transdisciplinar. Curitiba: Editora CRV; 2013. p. 69-86.

20. Patias ND, Siqueira AC, Dias ACG. Bater não educa ninguém! Práticas educativas parentais coercitivas e suas repercussões no contexto escolar. Educação e Pesquisa 2012; 38:981-96.

21. Andrade SSCA, Yokota RTC, Sá NNB, Silva MMA, Araújo WN, Mascarenhas MDM, et al. Relação entre violência física, consumo de álcool e outras drogas e bullying entre adolescentes escolares brasileiros. Cad Saúde Pública 2012; 28:1725-36.

22. Waikamp V, Serralta FB. Repercusiones del trauma en la infancia en la psicopatología de la vida adulta. Ciênc Psicol 2018; 12:137-44.

23. Cesaro BC, Bonamigo AW, Silva HTH, Oliveira $\mathrm{F}$. Changes in communication in children victims of violence: reflections for speech therapy. Distúrbios Comun 2016; 28:462-72.

24. World Health Organization. Child maltreatment: the health sector responds. Geneva: World Health Organization; 2017.

25. Dubowitz $\mathrm{H}$. The safe environment for every kid (SEEK) model: child healthcare professionals helping prevent child maltreatment. In: Dubowitz H, Bornes N, Tummala P, editors. World perspectives on child abuse. 12th Ed. Aurora: International Society for Prevention of Child Abuse and Neglect; 2014. p. 89-92.
26. Cezar PK, Arpini DM, Goetz ER. Registros de notificação compulsória de violência envolvendo crianças e adolescentes. Psicol Ciênc Prof 2017; 37:432-45.

27. Calza TZ, Dell'Aglio DD, Sarriera JC. Direitos da criança e do adolescente e maus-tratos. Revista da SPAGESP 2016; 17:14-27.

28. Pfeiffer L, Rosário NA, Cat MNL. Violência contra crianças e adolescentes: proposta de classificação dos níveis de gravidade. Rev Paul Pediatr 2011; 29:477-82.

29. Hohendorff JV, Habigzang LF, Koller SH. Violência sexual contra meninos: teoria e intervenção. Curitiba: Juruá Editora; 2014.

30. Viola TW, Salum GA, Kluwe-Schiavon B, Sanvicente-Vieira B, Levandowski ML, GrassiOliveira R. The influence of geographical and economic factors in estimates of childhood abuse and neglect using the Childhood Trauma Questionnaire: a worldwide meta-regression analysis. Child Abuse Negl 2016; 51:1-11.

31. Ribeiro IMP, Ribeiro AST, Pratesi R, Gandolfi L. Prevalence of various forms of violence among school students. Acta Paul Enferm $2015 ; 28: 54-9$.

32. Senado Federal. Constituição da República Federativa do Brasil, de 5 de outubro de 1988. http://www.planalto.gov.br/ccivil_03/cons tituicao/constituicao.htm (acessado em 14/ Ago/2018).

33. Brasil. Decreto no 99.710 , de 21 de novembro de 1990. Promulga a Convenção sobre os Direitos da Criança. Diário Oficial da União 1990; 22 nov.

34. Organização das Nações Unidas. Convenção sobre os direitos da criança. https://www.uni cef.org/brazil/convencao-sobre-os-direitosda-crianca (acessado em 31/Jan/2020).

35. Ormeno GR, Berlini LF. Maus-tratos infantis e Lei "Menino Bernardo". In: Gomide PIC, Staut Júnior SS, organizadores. Introdução à psicologia forense. Curitiba: Juruá Editora; 2016. p. 121-33.

36. Patias ND, Siqueira AC, Dell'Aglio DD. Imagens sociais de crianças e adolescentes institucionalizados e suas famílias. Psicol Soc 2017; 29: e131636.

37. Ministério da Saúde. Política Nacional de Redução da Morbimortalidade por Acidentes e Violências: Portaria MS/GM no 737 de 16 de maio de 2001. Brasília: Ministério da Saúde; 2001. (Série E. Legislação de Saúde, 8).

38. Brasil. Lei no 13.010, de 26 de junho de 2014. Altera a Lei no 8.069, de 13 de julho de 1990 (Estatuto da Criança e do Adolescente), para estabelecer o direito da criança e do adolescente de serem educados e cuidados sem o uso de castigos físicos ou de tratamento cruel ou degradante, e altera a Lei no 9.394, de 20 de dezembro de 1996. Diário Oficial da União 2014; 27 jun.

39. Organização das Nações Unidas. Relatório mundial sobre violência contra a criança. Geneva: ATAR Roto Presse SA; 2006. 
40. Ribeiro FB. Governo dos adultos, governo das crianças: Agentes, práticas e discursos a partir da "lei da palmada". Civitas 2013; 13:292-308.

41. Organização Mundial da Saúde. Relatório Mundial Sobre a Prevenção da Violência, 2014. São Paulo: Núcleo de Estudos da Violência, Universidade de São Paulo/Organização Mundial da Saúde; 2015.

42. Malterud K, Siersma VD, Guassora AD. Sample size in qualitative interview studies: guided by information power. Qual Health Res 2016; 26:1753-60.

43. Hohendorff JV, Postay AT, Habigzang LF, Koller SH. Parceria com a rede de atendimento no estudo da violência sexual infantil. Revista da SPAGESP 2017; 18:143-56.

44. BraunV, Clarke V. Using thematic analysis in psychology. Qual Res Psychol 2006; 3:77-101.

45. Ribeiro JML. Uso da palmada como ferramenta pedagógica no contexto familiar: mania de bater ou desconhecimento de outra estratégia de educação? Pesqui Prát Psicossociais 2012; 7:52-8.

46. Ricas J, Donoso MTV, Gresta MLM. A violência na infância como uma questão cultural. Texto Contexto Enferm 2006; 15:151-4.

47. Pinto LAPA, Colossi PM. Percepção materna acerca das práticas educativas coercitivas e a transmissão de modelos familiares. Contextos Clín 2017; 10:157-71.

48. Carmo PHB, Alvarenga P. Práticas educativas coercitivas de mães de diferentes níveis socioeconômicos. Estud Psicol (Natal) 2012; 17:191-7.

49. Pedersen JR, Grossi PK. O abuso sexual intrafamiliar e a violência estrutural. In: Azambuja MRF, Ferreira MHM, organizadores. Violência sexual contra crianças e adolescentes. Porto Alegre: Artmed; 2011. p. 25-34.

50. Dubowitz H, Kim J, Black MM, Weisbart C, Semiatin J, Magder LS. Identifying children at high risk for a child maltreatment report. Child Abuse Negl 2011; 35:96-104.

51. Ricas J, Donoso MTV. Maus tratos na infância: reflexões. REME Rev Min Enferm 2006; 10:306-10.

52. Ribeiro FB. A proibição legal de castigos físicos na infância: alguns contrastes entre Brasil, Uruguai e França. Desidades 2016; 10:19-28.

53. Njaine K, Assis SG, Gomes R, Minayo MCS. Redes de prevenção à violência: da utopia à ação. Ciênc Saúde Colet 2006; 11 Suppl:1313-22.

54. Mourão L, Monteiro ACF, Viana VR. A influência do desenvolvimento profissional e da identificação organizacional na satisfação no trabalho. Psico 2014; 45:198-208.
55. Paquay L, Wouters P, Van Nieuwenhoven C. A avaliação, freio ou alavanca do desenvolvimento profissional? In: Paquay L, Wouters P, Van Nieuwenhoven C, organizadores. A avaliação como ferramenta do desenvolvimento profissional de educadores. Porto Alegre: Penso; 2012. p. 13-39.

56. Faraj SP, Siqueira AC, Arpini DM. Rede de proteção: $\mathrm{O}$ olhar de profissionais do sistema de garantia de direitos. Temas Psicol 2016; 24:727-41

57. Coelho Junior FA, Mourão L. Suporte à aprendizagem informal no trabalho: uma proposta de articulação conceitual. RAM Revista de Administração Mackenzie 2011; 12:224-53.

58. Deslandes S, Campos D. A ótica dos conselheiros tutelares sobre a ação da rede para a garantia da proteção integral a crianças e adolescentes em situação de violência sexual. Ciênc Saúde Colet 2015; 20:2173-82.

59. Silva PA, Lunardi VL, Meucci RD, Algeri S. Protection of children and adolescents victims of violence: the views of the professionals of a specialized service. Invest Educ Enferm 2018; 36(3).

60. Ministério da Saúde. Portaria no 1.356 de 23 de junho de 2006. Institui incentivo aos estados, ao Distrito Federal e aos municípios para a Vigilância de Acidentes e Violências em Serviços Sentinela, com recursos da Secretaria de Vigilância em Saúde (SVS). http:// www.saude.sp.gov.br/resources/cve-centrode-vigilancia-epidemiologica/areas-de-vigi lancia/doencas-cronicas-nao-transmissiveis/ observatorio-promocao-a-saude/portarias/ portaria_gm1356_2006.pdf (acessado em 07/ Mar/2020).

61. Ministério da Saúde. Portaria no 204, de 17 de fevereiro de 2016. Define a Lista Nacional de Notificação Compulsória de doenças, agravos e eventos de saúde pública nos serviços de saúde públicos e privados em todo o território nacional, nos termos do anexo, e dá outras providências. Diário Oficial da União 2006; 18 fev.

62. Verch K. Primeira Infância Melhor. Transformando a atenção aos primeiros anos de vida na América Latina: desafios e conquistas de uma política pública no sul do Brasil. s.l.: Banco Interamericano de Desenvolvimento; 2017. 
Abstract

Although many countries have passed laws to ban the use of physical violence for purported educational purposes, the World Health Organization has stated that the enforcement of such laws is still a challenge. The study thus aimed to verify whether networks in Brazil for the protection and care of children and adolescents at risk are organized to enforce the Menino Bernardo Law (Law n. 13,010/2014). Using a qualitative and exploratory approach, the study interviewed 16 professional staff members from different services in these networks in a city in the state of Rio Grande do Sul, Brazil. The data collected in the interviews were analyzed with inductive thematic analysis, resulting in four themes: (1) culturally accepted practice; (2) ignorance versus awareness of the law; (3) shortage of networks; and (4) enforcement strategies. Taken as a whole, the four themes point to non-enforcement of the Menino Bernardo Law by the networks for protection and care of children and adolescents at risk in the municipality where the study was carried out. This revealed social acceptance of resort to violence in raising children and adolescents; ignorance of the Menino Bernardo Law by the staff; and fragmentation between professional staff and the networks' services. It is thus necessary to strengthen institutional resources and staff training to improve professional development and the networks' services.

Child Abuse; Child Advocacy; Child Rearing; Parent-Child Relations; Legislation as Topic

\section{Resumen}

A pesar de que muchos países estén implementando leyes que prohíben el uso de la violencia física, como supuesta práctica educativa, la Organización Mundial de la Salud indica que el cumplimiento de esas leyes todavía es un desafío. El objetivo fue, por tanto, verificar si las redes de protección $y$ de atención a niños y adolescentes en situación de riesgo están organizadas para hacer cumplir la Ley Menino Bernardo (Ley no 13.010/2014). Mediante un enfoque cualitativo y exploratorio, se entrevistaron a 16 profesionales de diferentes servicios de esas redes en un municipio del interior de Río Grande do Sul, Brasil. Los datos recogidos en las entrevistas se analizaron mediante un análisis temático de tipo inductivo, dando como resultado cuatro temas: (1) práctica aceptada culturalmente; (2) (des)conocimiento de la ley; (3) carencias de las redes y (4) estrategias de cumplimiento. Tomados en conjunto, los cuatro temas apuntan al no cumplimiento de la Ley Menino Bernardo por parte de las redes de protección y atención a niños $y$ adolescentes en situación de riesgo, en el municipio donde se realizó la investigación. Se evidenció, por ello, la aceptación social de la violencia en la educación de niños y adolescentes; el desconocimiento de la Ley Menino Bernardo por parte de los profesionales, asi como la fragmentación entre los profesionales y los servicios de las redes. Ante esta situación, es necesario aumentar la inversión para el fortalecimiento de los recursos institucionales, así como para la formación, teniendo como meta el desarrollo profesional y la cualificación de los servicios de las redes.

Maltrato a los Niños; Defensa del Niño; Crianza del Niño; Relaciones Padre-Hijo; Legislación como Asunto
Recebido em 03/Out/2019

Versão final reapresentada em 11/Mar/2020

Aprovado em 20/Mar/2020 